\title{
DATA-DRIVEN DESIGN AUTOMATION FOR PRODUCT- SERVICE SYSTEMS DESIGN: FRAMEWORK AND LESSONS LEARNED FROM EMPIRICAL STUDIES
}

\author{
Machchhar, Raj Jiten; \\ Bertoni, Alessandro \\ Blekinge Institute of Technology
}

\begin{abstract}
The digitalization era has brought about unprecedented challenges for the manufacturing industries, pushing them to deliver solutions that encompass both product and service-related dimensions, known as Product-service Systems. This paper presents a number of lessons learned in the process of integrating the analysis of operational data as decision support in engineering design based on the empirical studies from two Swedish manufacturing companies operating in the construction machinery sector. The paper highlights the need to consider a five-dimensional perspective when collecting and analyzing data, encompassing data from the product, the service, the environment, the infrastructure, and the humans involved. Finally, a conceptual framework for data-driven design automation of Product-service Systems is proposed by leveraging the use of these data, introducing the concept of a Product-Service System Configurator as an enabler of design automation. The implementation of the proposed framework on multiple case studies in different industrial contexts shall be considered as the next step of the research.
\end{abstract}

Keywords: Product-Service Systems (PSS), Data-driven Design, Design Automation, Systems Engineering (SE), Decision making

\section{Contact:}

Machchhar, Raj JIten

Blekinge Institute of Technology

Mechanical Engineering

Sweden

raj.jiten.machchhar@bth.se 


\section{INTRODUCTION}

It is nowadays widely recognized that the digitalization wave has nurtured an era of unprecedented technological innovation and has impacted a large majority of manufacturing industries. The industrial pace of innovation is often dependent on the complexity of the supersystem (Kossiakoff and Sweet, 2003) (i.e. the "machine" or "asset" as a system plus the "infrastructure" as supporting systems) and the supply chain architecture. In traditional industries with high capital commitment, long product lifecycle, and complex supply chain, such transformation is relatively slower compared to leaner organizations. Complex development projects are guided by established systems engineering processes highly relying on modeling and simulations to guide design decisions (Kossiakoff and Sweet, 2003). In such a context, years of development have delivered increasingly more accurate mechanical simulations, allowing for multidisciplinary design optimization (MDO) to support design decisions, counting on the use of surrogate modeling and design automation (DA) (Amadori, 2012). However, such established engineering design approaches do not completely fulfill the potential of delivering radically new and innovative systems, especially in contexts in which digital technologies allow the concurrent design, management, and delivery of solutions that encompass both product and service-related dimensions. Academic literature often describes such a new scenario as a transition from traditional product development (PD) to the development of Product-service Systems (PSS) (Baines et al., 2009). In such a context, during the early stages of design, it is extremely difficult to predict how a future PSS will behave: there is no availability of a generic platform to digitally represent a PSS as a solution. Thereto, running MDO or DA is far from being a possibility.

The research presented in this paper is based on the identification of the shortcomings of the current engineering practices in designing PSS responding to the need for innovation driven by digitalization. In particular, the paper focuses on the potential integration of data-driven design support in the context of MDO and DA for PSS development in systems engineering projects.

With such a purpose, the paper builds on previous research on the data-driven design in the construction industry (e.g. Bertoni et al., 2017; Chowdhery and Bertoni, 2018) and describes the findings collected through a participatory action research approach (Whyte, 1989) in two well-established Swedish construction machinery companies. Based on the empirical research the paper has a twofold objective:

- Firstly, it presents a number of lessons learned on using operational data as decision support for early-stage design decisions. Those encompass the process of collecting, organizing, integrating, and utilizing machine usage data in the engineering design process.

- Secondly, it proposes a framework for data-driven DA of PSS, bringing arguments to the need to reconsider the concept of "design automation" in the context of a PSS by leveraging the use of data from a five-dimensional perspective, that is, considering the product, the services, the environment, the infrastructure, and the humans involved.

The structure of the paper is the following: section 2 briefly describes the research approach based on action research, section 3 provides an overview of the relevant literature on the topic, section 4 describes the lessons learned while section 5 details the proposed conceptual framework. Finally, section 6 positions the current work in the existing literature and draw the final conclusions.

\section{RESEARCH APPROACH}

In the frame of the participatory action research effort, data have been gathered during four years by means of open-ended and semi-structured interviews, company presentations, and concurrent development of demonstrators. The lessons learned described in the paper can be seen as results of the first descriptive study in the frame of the Design Research Methodology (Blessing and Chakrabarti, 2009), while the proposed framework can be seen as the results of an initial prescriptive study.

Several reasons have led to the identification of the construction machinery context as the most suitable for the investigation of data-driven DA for PSS. Such industry has engaged a long journey toward the transformation to fully electrical and autonomous vehicles (Frank, 2019). Examples of machine prototypes running fully electrical and in autonomy have been presented by major providers of construction machinery worldwide. A key challenge in such transition is the capability to design and developed a new, effective, and efficient system based on autonomy and electromobility in an industrial sector that has traditionally been conservative, with a high focus on pure mechanical performances. In such a context, the transition toward PSS, thus toward ownership retainment, has opened possibilities to 
collect data that are higher than before, thus making it possible to formulate stronger data-driven inferences. Additionally, the working environment for construction machinery is more controlled, restricted, and predictable with less possibility of unexpected events (compared, for instance, to autonomous cars driving in an urban environment), making it more suitable for data collection from machines and the surroundings. These characteristics made the construction machinery context to be identified as a suitable research environment and potentially a good testbed for the research.

\section{RELEVANT LITERATURE}

\subsection{Design automation and product-service systems development}

Computer-aided Design (CAD) tools are widely accepted in many industries today with their extended support to the PD process as enablers of model-based engineering. With several activities in developing a CAD model identified as routine-like (Stokes and MOKA Consortium, 2001), researchers have increasingly acknowledged design automation (DA) as a method to reduce this repetitiveness by automating the mundane design tasks. In the context of product and CAD, one of the prominent methods is geometry-based DA (Amadori, 2012) essentially utilizing CAD templates (Tarkian et al., 2012) to configure different variants of designs. Amadori (2012) shows how geometrybased DA has enabled CAD tools to serve as framework integrators for multi-disciplinary analysis in complex product or system development projects, highlighting that geometry-based DA needs external decision support to choose a design configuration. The work in this paper refers to DA in line with Amadori (2012) as a means to automate (partially or fully) the design tasks, essentially configuring a PSS concept to expedite the PSS development process. In the context of service and CAD, literature highlights service CAD (Arai and Shimomura, 2004) as one of the popular tools to represent services. Researchers (e.g. Nemoto et al., 2012) have also proposed a knowledge base comprising of different service modules that can potentially enable DA. Knowledge-based engineering (KBE) is identified as one of the methods to enable DA utilizing existing knowledge in the engineering design process. As Rocca (2012) states, KBE is a technology based on KBE systems that capture, store, and formalize design knowledge that emerges in a design process for its systematic reuse to enable DA and MDO. Van der Velden et al. (2012) state that KBE enables automation of processes in a PD lifecycle, resulting in a reduction in time and cost, and capture and retain knowledge for later use. Several researchers have applied DA frameworks in their respective fields. Amadori et al. (2012) applied CAD templates to design transport aircraft, industrial robots, and micro air vehicles by enabling MDO. Frank et al. (2014) applied DA for customer-specific goods via a product configurator. Their work was demonstrated by two case studies, arguing that such configurators are generic and can be applied in other fields too. More recently, Poot et al. (2020) integrated sales and design via DA frameworks, where they demonstrated that all the PD phases from a customer need to production preparation can be automated via KBE systems. Thereto, DA has been rigorously explored for product design, but sparse evidence exists in literature when the development is an integrated outlook (Isaksson et al., 2009), i.e. a combination of product and service. Johansson et al. (2017) highlighted a possibility to incorporate PSS-based business models in their DA framework. They envisaged the co-creation of solutions with customers, but no evidence of the existence of such a framework is provided. Certainly, for the integrated outlook, KBE systems need to be dynamic, i.e. more flexible and adaptive to incorporate 'servitization' of products and 'productization' of services.

Concerning the development process of a PSS, literature highlights several PSS design methodologies that could be adapted and extended for DA. One such framework was illustrated by Song and Sakao (2017), where the PSS design comprises four phases: requirement identification, conflict resolution, modularization, and configuration. Requirement identification refers to deriving functional requirements (Isaksson et al., 2009) from customer needs. Conflict resolution implies arriving at a consensus for contrasting design variables and constraints. Modularization is a known term in the PD process, broadly as the creation of specific modules relatively independent of each other to achieve the desired function (Ulrich and Eppinger, 2012). Finally, configuration implies the selection of proper modules for the given functional requirements.

\subsection{Data-driven design}

Data is increasingly considered as a resource in many industries and data mining has emerged as a discipline for discovering previously unseen knowledge in datasets. This practice of using data-driven 
models to improve products, services, and systems, has found its application in many industrial sectors, right from creating transparency to innovating the next generation of solutions (Manyika et al., 2011). Kusiak (2006) highlighted the importance of a data-driven paradigm in manufacturing and service industries emphasizing that the quality of decision support depends on the quality of knowledge extraction from datasets. Kulin et al. (2016) recognized two uses of such data-driven models, one is to better understand the inferred system to gain deep and valuable insights about it and the other is to generate extrapolated data that mimic the inferred system.

With advancements in data mining techniques, data-driven models have found their position in predicting outcomes and support engineering design beyond human cognition where decisions are usually based on experience. Translating customer needs has been one of the major focuses of datadriven design (e.g. Barnes and Lillford, 2009; Chaklader and Parkinson, 2017), although some authors have demonstrated the use of product or system usage data to aid engineering design. Shin et al. (2015) supported design modification by fixing design parameters based on the identified suspicious field data from the working status of the product. Bertoni et al. (2017) demonstrated the use of operational data to complement experience-based decisions. Ma et al. (2017) proposed a design improvement approach based on the time-dependent product usage data, where they improved design parameters in three steps: creating a function model, assessing the function degradation, and identifying the design parameters to be modified. However, in all these studies, no reference to automating the design solution was found. Sakao and Neramballi (2020), in their literature review, highlight that big data analytics, enabled by exploiting the product and service usage data, has a tremendous potential to assist the concept development stage of a PSS, right from functional unit definition to a viable solution selection. However, Bertoni (2020) highlighted that the application of data analytics in the concept development stage has been dominantly based on data from customer's perception of use rather than the actual product, service, or system usage data, the latter being largely unexplored. Thus, data-driven design automation from an operational data viewpoint in the concept development stage is largely unexplored and the opportunities are two-fold, one is to extend the current research by leveraging the use of operational data in the concept development stage and the other is automating the repetitive design tasks by formalizing this knowledge in a decision support system.

\section{LESSONS LEARNED FROM THE CONSTRUCTION MACHINERY INDUSTRY}

The aforementioned construction machinery industries are facing distinct challenges in their quest of providing "functions" instead of "products", rendering a situation for these industries to offer PSS as a solution. To envisage the complete picture (referred to as "operational frame") of such a system operating in its surroundings, it is crucial to first define the "boundary" of the inferred system. Systems engineering proposes development control (Kossiakoff and Sweet, 2003) as one of the criteria to qualify within this boundary. From this viewpoint, all the entities (natural or man-made), that the system interacts with, that are beyond the scope of the development project fall outside the boundary of the system. A similar analogy is made by Haberfellner et al. (2019) referring to the inferred system as the "system of interest" with "interior" and "exterior" boundaries. From this viewpoint, humans, though considered to be an essential aspect for the operation of a system are external. Thus, an operational frame consists of three key elements (Kossiakoff and Sweet, 2003): the system, the externalities, and their interactions. The influence of the externalities on the system has been considered by many researchers, such as (Belk, 1975) emphasizing "situational characteristics" and (Green et al., 2005) considering "usage context". From their work, the externalities can be mainly categorized into the environment, infrastructure, and human.

Operational data is a virtual manifestation of the interactions between the system and the externalities. Mining this data to create data-driven models and learning from those models can support the early design trade-offs, bring consensus between different stakeholders, and facilitate the transition of these industries towards autonomy and electrification. But several challenges emerged during the empirical studies that need to be addressed before any meaningful inferences can be made from this data. These challenges and the lessons learned are summarized below and also illustrated in Figure 1:

- Data collection: Empirical studies highlighted an evident need to plan the collection of operational data to avoid the following problems. Firstly, data in manufacturing industries is often collected without a specific purpose, leading to a plethora of unnecessary data. A purposeful collection of 
data would aid in trimming the non-contextual data. Secondly, manufacturing industries often have lesser competence in information technology and prefer outsourcing data collection to a third party. As a result, companies might not own complete rights to the collected data. Thus, the ownership of the data must be planned, where the level of accessibility to the collected data from the third parties is on the higher side. Thirdly, the right timeframe and format need to be planned as well for the collection of data. The right timeframe has two aspects, one is the right period and the other is the right sampling rate, chosen cautiously to avoid information overload. Right format implies using the formats that can be easily accessed and manipulated by the user. Choosing the right timeframe and format are subjective matters, much dependent on the competencies of the people in the industry, tools available at hand, the time scale over which data-driven inferences need to be made, and again, the purpose of collecting data.

- Data organization: Data organization relates to the right practice of annotating and storing the collected data. The collected data must be archived such that the origin of data can be traced, essentially by utilizing annotations. Functional teams in the manufacturing industries usually have their own terminologies to annotate entities. Thus, a common basis for annotations must be established if there is a likelihood that other teams might access this data in the future. Also, it is worth highlighting that the annotation does not necessarily mean comprehensibility. To illustrate with a simple example, one of the common practices to denote time in sampled data is to use Unix Epoch Time (Matthew and Stones, 2008), i.e. counting total seconds elapsed from 1st January 1970, to avoid confusion in time zones. Such an arbitrary 10-digit number (as of today) is impractical to indicate the time to humans, even though it is annotated as "time". Thus, measures need to be taken to denote one-to-one correspondence between Unix Epoch Time and the current zonal time, leading to a recommendation of having measures taken to make data more understandable to humans. With these practices, the unknown meaning of the data is inherently documented, and interpreting it does not become a challenge, especially when key people in the industry leave.

- Data integration: In a complex system, the challenge in developing a new solution is the identification of how the design variables at the component-level (or the bottommost level) affect the performance at the system-level (or the topmost level), mainly because of the long hierarchical supply chain architecture. Deriving a correlation between these component-level design variables (or design variables) to the system-level performance (or performance) needs a comprehensive understanding of the overall behavior of the system in its surrounding, identifying the implications at each level. Thereto, a purposefully collected well-organized operational data is the first step towards creating a virtual representation of the operational frame to understand it better. It is important to acknowledge that operational data encompasses a multidimensional viewpoint, comprising of data segregated into five dimensions: product, service, environment, infrastructure, and human (refer to Figure 2). As mentioned previously, operational data is a result of the interactions between the system and the externalities, and thus, the categories may not have any substantial significance independently. The above segregation comes for the design team's interpretation convenience. The way to knowledge from operational data is long and complex, and the challenge for the design team is to integrate operational data as data-driven models to form the links between the design variables and the performance.

- Data utilization: Often, manufacturing industries struggle in the proper utilization of data, the root cause can be attributed to prediction accuracy and reliability of data-driven models. Prediction accuracy has two loose ends, one is the input data and the other is the performance measure of the learning algorithm used to develop the data-driven model. The performance measure of learning algorithms is well addressed in the literature (Sokolova and Lapalme, 2009), and there has been a notable improvement over the years in this domain. The challenge lies in providing the right data, averting the design team from misinterpreting precision as accuracy. Assuming that the integration of purposefully collected, well-organized, five dimensions of operational data solve the accuracy problem by eliminating noise and redundancy, the reliability problem is still unsolved. The challenges in this context are more related to human cognition. Datadriven models are often perceived as a "black box", to what Freitas (2014) elaborates that predicting accuracy has been the major criterion to evaluate the performance of a model, and interpretability is largely ignored. Data-driven models need to be well-understood by the design team and provide some level of confidence in the design team, in cases of high uncertainty. The 
level of confidence, in this context, implies that the inferences made from data-driven models need to be clearly expressed in terms of completeness and maturity of data, thus laying a firm grounding to the inferences. Such an approach enables the design team to rely lesser and lesser on experiencebased decisions, refraining them from "extremeness aversion", as highlighted by Bertoni (2018).

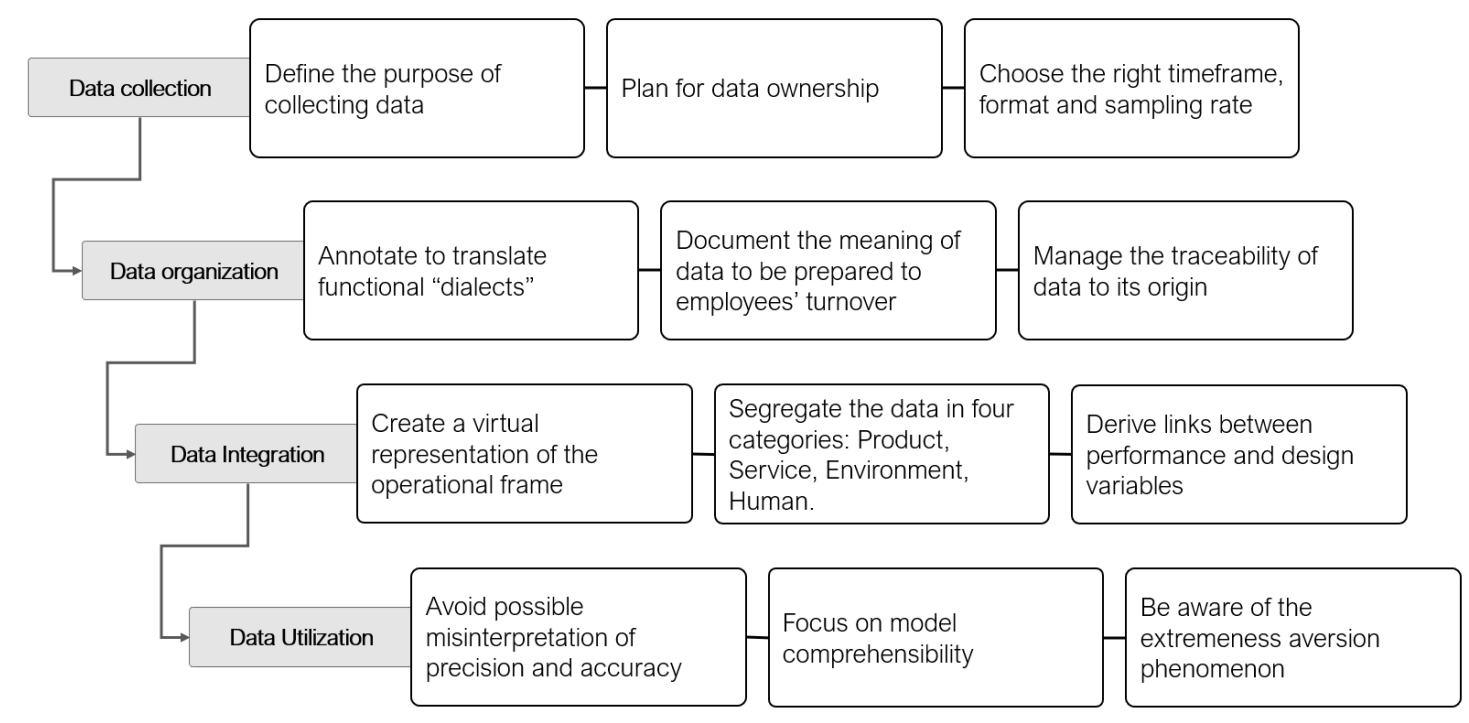

Figure 1. Lessons learned in using operational data as design supports

\section{A FRAMEWORK FOR THE TRANSITION TO DATA-DRIVEN DESIGN AUTOMATION IN PSS}

A representative framework to automate a PSS solution is demonstrated in Figure 2. The need to develop a PSS arises from identifying the customer needs that are translated into functional requirements. Notably, as Figure 2 illustrates, the loop from identifying the customer needs to deliver the solution in the form of products and/or services to the customer can indeed be completed without any external decision support, certainly for much simpler products. However, for complex systems, the translation of customer needs into functional requirements may not be interpreted as the development of a physical entity, mainly because the physical entity is only a part of the complete solution. The development of a solution for such functional requirements encourages the industries to develop a PSS, where the challenge lies in the essence of critically weighted hybrids of products and services that create value for the customer. Thus, aligning with Isaksson et al. (2009), the solution provided to the customer, in the context of a PSS, can be in the form of a product but be purely service-oriented.

When such a PSS is based around an established product architecture, many inferences can be made about the system by analyzing the behavior of the system in its surrounding. The growing availability of data creates an opportunity to evaluate the performance of the system against the objective and learn about the system early in the design phase. The five dimensions of data emerging as a consequence of interactions between the system and the externalities have been briefly described in section 4, and are visualized on the left-hand side of Figure 2. These data, stored in the database (at the center of Figure 2), is retrieved for the development of a new PSS by populating data-driven models. The key to formulating meaningful data-driven models lies in the "isolated integration" of selected datasets. Here, isolated integration refers to the notion of creating a boundary between relevant data sets and noise to depict a specific characteristic of the inferred system/subsystem. Simply put, isolation refers to eliminating the redundant data and integration refers to combining all the relevant data to improve prediction accuracy. Thereto, Lützenberger et al. (2016) highlight that statistical functions have a crucial potential role in such filtration. Such data-driven models, that can mimic the inferred system, are necessary to understand the behavior of the system at all subsystem-levels. Adaptive control is one of the known applications of operational data usage, where data is analyzed in real-time to take corrective actions. The inferred application operational data here is different, in the sense that the timeframe of learning could be days, months, or even years instead of mere seconds. To illustrate, a "vibratory" drum is a crucial design aspect of the road compaction machine. The vibration frequency setting that must be ideally used at a particular instance is an application of adaptive control, while the 
settings of vibration frequencies that should be present in a machine is an application of inferences made from the data-driven models. Towards this end, data-driven models may not be the sole and selfsufficient choice for decision-making. Researchers (e.g. Wright and Davidson, 2020) have argued that data-driven models are reliable strictly in the frame of references, and their generalizability is often questionable. However, when the timeframe for making decisions is longer, there is the possibility to complement the decision-making activity with other assessment models, as shown in the bottom right corner of Figure 2. The nature of such models is context-dependent; they can be experience-based models and/or physics-based models. Often, the choice of which models to consider in decision making is dependent by the desired level of data reliability, or knowledge maturity as defined by Johansson et al. (2011) needed at the different stages of the PD process.

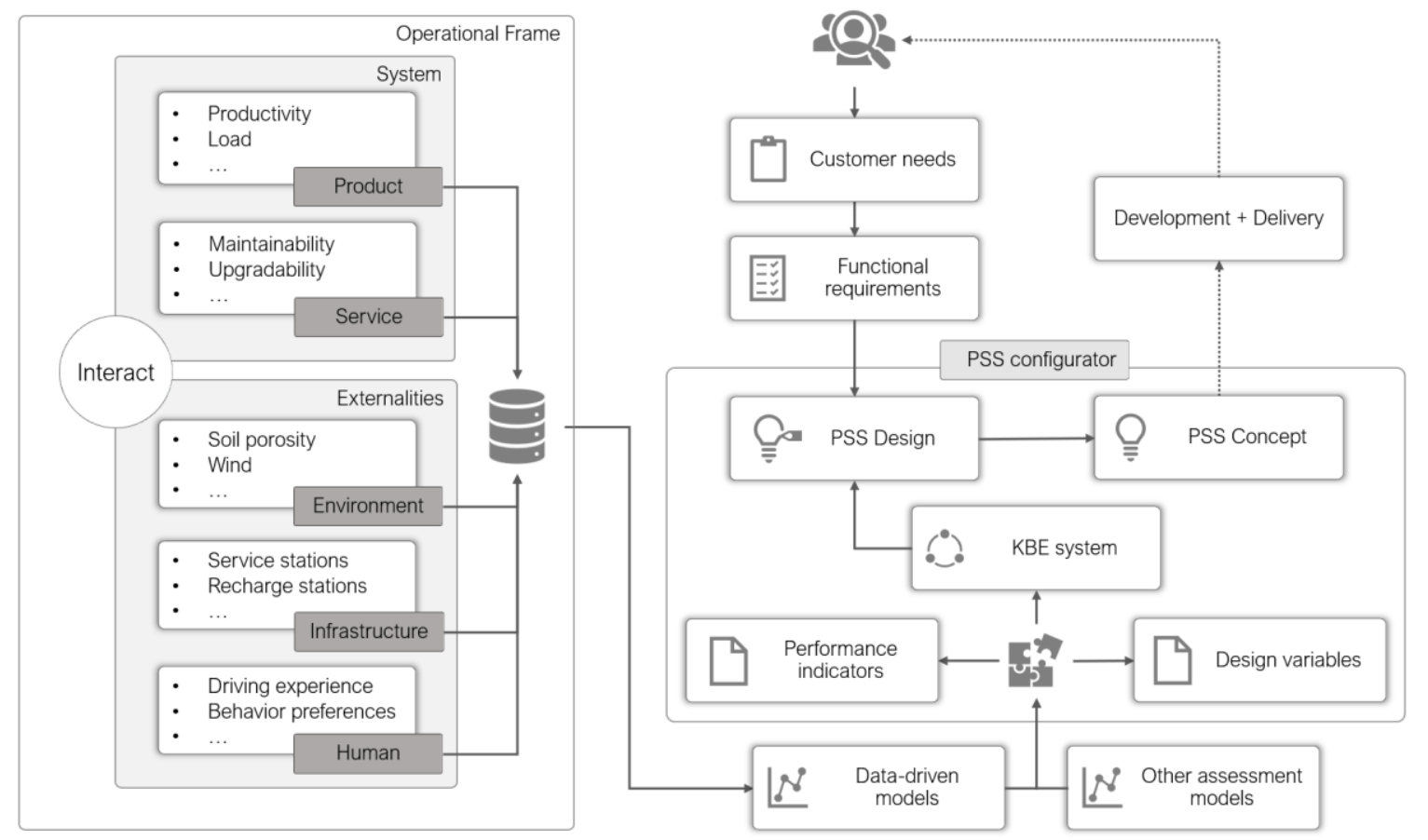

Figure 2. A framework for data-driven design automation of product-service systems

From a PSS viewpoint, inside the boundaries of the PSS configurator in Figure 2, the amalgam of data-driven models and other assessment models creates the needed link between the design variables and the performance for the PSS design. These design variables comprise of the tangible productrelated variables and the intangible service-related variables. The product-related variables influence the PSS design by leveraging knowledge about the collaborative functionality of mechanical entities as well as electrical, electronic, and software entities to achieve the desired performance requirements. The service-related variables influence the PSS design by adding dimensions like repairability, replaceability, upgradability, circularity, etc. at the provider's ends and dimensions like software updates, training, personalization, etc. at the consumer's end. A similar mapping of performance to design variables, enabled by operational data, was demonstrated by Lützenberger et al. (2016) where they envisage co-designing the PSS from product and service-related variables.

Once a satisfactory mapping of performance to design variables is achieved, KBE systems serve as a kernel to formalize this knowledge to reuse it in the design process. Referring to the framework illustrated by Song and Sakao (2017), PSS design, in this context, consists of three phases: conflict resolution, modularization, and configuration, and the modules essentially comprise of product and service attributes. Constrained exploration and optimization are often important to achieve better and reliable results, analyzing different modules and their integration to achieve the objective. Towards this end, the KBE system enables DA of various potential configurations of the PSS to allow exploration and optimization. Although, one must not perceive exploration and optimization as the sole purpose of KBEenabled DA. A PSS concept (to the right of Figure 2) quickly realized from previous learnings and brought to the discussion forum between various stakeholders also falls within the application of KBE enabled DA. Principally speaking, the role of such a system is not to substitute the design team in making decisions and generate a "one-click" solution for a design problem, rather support them by 
increasing awareness and helping in making conscious decisions. Nonetheless, the design team must confide in the models and their collaboration to predict an outcome, and that is when a potential opportunity is truly utilized, and a meaningful concept is generated. Furthermore, the PSS concept may not be interpreted as the final solution. The dotted lines in Figure 2 denote the fact that there are certainly further steps before the solution is delivered to the customer such as detailed engineering, testing, production, etc. depending on the application. In the context of a PSS, implementation, and monitoring also become vital elements of solution delivery, as highlighted by Sala et al. (2020).

The significance of the "PSS configurator" in Figure 2 is that the interface representing a PSS solution digitally needs to account for many more aspects than traditional CAD geometry. Typically, a PSS configurator: 1) needs to represent the data from the five dimensions, that is the product, the services, the environments, the infrastructures, and the humans involved, 2) allows the collaboration of other assessment models to enhance the decision support, 3) is able to represent the PSS right from the component-level up to the system-level, 4) enables the establishment of the vital links between the performance and the design variables, 5) can formalize and use this knowledge to explore the design space, 6) is capable of presenting an optimized solution in the design space with indications on level of confidence, and 7) is able to autonomously configure the designs based on the inputs. Here, the opportunity to design the product-service hybrid as a system extends beyond mechanical products to include sensors, actuators, networks, interfaces, software, programming languages, and ultimately consider the "human aspect" in a dynamic environment.

\section{CONCLUDING REMARKS}

The paper has presented some lessons learned in the construction machinery industry concerning the use of operational data as decision supports in engineering design. The lessons learned have been divided into four categories, namely collection, organization, integration, and utilization of data. Based on the lessons learned and the challenges identified during the empirical studies, the paper has illustrated the first version of a conceptual framework for the Data-driven Design Automation of Product-service Systems. The findings are based on the results of empirical studies in collaboration with two companies operating in a similar business in the construction machinery sector. Many unknowns still need to be discovered to allow the generalization of the findings to other industrial sectors.

The lessons learned in data collection, organization, integration, and utilization have been observed and formalized in the process of building meaningful data-driven models to support engineering design decision-making. An analogy in terms of data-driven decision making has been made by (Miller and Mork, 2013) providing a framework illustrating the important steps from collecting raw data to making informed data-driven decisions, identifying data sharing as one of the major obstacles in data-driven decision making. The proposed framework stressed the need to consider the five-dimensional perspectives of operational data to design solutions for functional requirements, encompassing the product, the services, the environments, the infrastructure, and the humans involved. Furthermore, it stresses the need for a PSS configurator as an enabler of design automation, a topic that was not addressed by previous frameworks to integrate products and services (e.g. Sundin et al., 2009; Sakao et al., 2020). Positioning the proposed framework in respect to the literature on PSS methodology, the work aligns with part of the 4DPSS methodology for PSS design and delivery proposed by Sala et al. (2020) and can be seen as a complementary step towards a more detailed definition of a data-driven concept generation and design phase encompassing the possibility of design automation.

In conclusion, the presented work contributes to the engineering design practices by sharing the lessons learned during the transition to data-driven design in complex systems, and to the academic discussion of Design Automation in the context of Product-service Systems. Although the empirical data collection being performed only in the construction machinery industry, literature shows that the underlining challenges are not unique to such industry but are shared in other industrial sectors as well, giving promising insights for the future generalization of the findings in different industrial contexts.

The implementation of the proposed framework on multiple case studies in different industrial contexts in the frame of systems engineering projects shall be considered as the next research step. 


\section{ACKNOWLEDGMENT}

The research has received financial support from the Swedish Knowledge and Competence Development Foundation through the Model-Driven Development and Decision Support research profile at Blekinge Institute of Technology.

\section{REFERENCES}

Amadori, K. (2012), Geometry Based Design Automation: Applied to Aircraft Modelling and Optimization, Doctoral thesis, comprehensive summary, Linköping University Electronic Press, Linköping.

Amadori, K., Tarkian, M., Ölvander, J. and Krus, P. (2012), "Flexible and robust CAD models for design automation", Advanced Engineering Informatics, Vol. 26 No. 2, pp. 180-195. https://doi.org/10.1016/j.aei.2012.01.004

Arai, T. and Shimomura, Y. (2004), "Proposal of Service CAD System - A Tool for Service Engineering -", CIRP Annals, Vol. 53 No. 1, pp. 397-400. https://doi.org/10.1016/S0007-8506(07)60725-2

Baines, T.S., Lightfoot, H.W., Benedettini, O. and Kay, J.M. (2009), "The servitization of manufacturing: A review of literature and reflection on future challenges", Journal of Manufacturing Technology Management, Emerald Group Publishing Limited, Vol. 20 No. 5, pp. 547-567. https://doi.org/10.1108/17410380910960984

Barnes, C. and Lillford, S.P. (2009), "Decision support for the design of affective products", Journal of Engineering Design, Taylor \& Francis, Vol. 20 No. 5, pp. 477-492. https://doi.org/10.1080/09544820902875041

Belk, R.W. (1975), "Situational variables and consumer behavior", Journal of Consumer Research, The University of Chicago Press, Vol. 2 No. 3, pp. 157-164.

Bertoni, A. (2018), "Role and Challenges of Data-Driven Design in the Product Innovation Process", Vol. 51, presented at the 16th IFAC Symposium on Information Control Problems in Manufacturing - INCOM 18, Elsevier, pp. 1107-1112.

Bertoni, A. (2020), "Data-driven design in concept development: systematic review and missed opportunities", Proceedings of the Design Society: DESIGN Conference, Vol. 1, Cambridge University Press, pp. 101-110. https://doi.org/10.1017/dsd.2020.4

Bertoni, A., Larsson, T., Larsson, J. and Elfsberg, J. (2017), "Mining data to design value: A demonstrator in early design", DS 87-7 Proceedings of the 21st International Conference on Engineering Design (ICED 17) Vol 7: Design Theory and Research Methodology, Vancouver, Canada, 21-25.08. 2017, pp. 021-029.

Blessing, L.T. and Chakrabarti, A. (2009), DRM: A Design Reseach Methodology, Springer.

Chaklader, R. and Parkinson, M.B. (2017), "Data-Driven Sizing Specification Utilizing Consumer Text Reviews", Journal of Mechanical Design, American Society of Mechanical Engineers Digital Collection, Vol. 139 No. 11. https://doi.org/10.1115/1.4037476

Chowdhery, S.A. and Bertoni, M. (2018), "Modeling resale value of road compaction equipment: a data mining approach", IFAC-PapersOnLine, Vol. 51 No. 11, pp. 1101-1106. https://doi.org/10.1016/j.ifacol.2018.08.457

Frank, G., Entner, D., Prante, T., Khachatouri, V. and Schwarz, M. (2014), "Towards a Generic Framework of Engineering Design Automation for Creating Complex CAD Models", International Journal of Advances in Systems and Measurements, Vol. 7, pp. 179-192.

Frank, M. (2019), A Step Towards the Design of Collaborative Autonomous Machines : A Study on Construction and Mining Equipment, Licentiate thesis, comprehensive summary, Blekinge Institute of Technology Licentiate Dissertation Series, Blekinge Tekniska Högskola, Karlskrona.

Freitas, A.A. (2014), "Comprehensible classification models: a position paper", ACM SIGKDD Explorations Newsletter, Vol. 15 No. 1, pp. 1-10. https://doi.org/10.1145/2594473.2594475

Green, M.G., Tan, J., Linsey, J.S., Seepersad, C.C. and Wood, K.L. (2005), "Effects of Product Usage Context on Consumer Product Preferences", presented at the ASME 2005 International Design Engineering Technical Conferences and Computers and Information in Engineering Conference, pp. 171-185. https://doi.org/10.1115/DETC2005-85438

Haberfellner, R., De Weck, O., Fricke, E. and Vössner, S. (2019), Systems Engineering: Fundamentals and Applications, Springer.

Isaksson, O., Larsson, T.C. and Rönnbäck, A.Ö. (2009), "Development of product-service systems: challenges and opportunities for the manufacturing firm", Journal of Engineering Design, Taylor \& Francis, Vol. 20 No. 4, pp. 329-348. https://doi.org/10.1080/09544820903152663

Johansson, C., Hicks, B., Larsson, A. and Bertoni, M. (2011), "Knowledge Maturity as a Means to Support Decision Making During Product-Service Systems Development Projects in the Aerospace Sector", Project Management Journal, Project Management Institute, Vol. 42 No. 2, pp. 32-50. https://doi.org/10.1002/pmj.20218

Johansson, C., Larsson, T. and Tatipala, S. (2017), "Product-Service Systems for Functional Offering of Automotive Fixtures: Using Design Automation as Enabler", Procedia CIRP, Vol. 64, pp. 411-416. https://doi.org/10.1016/j.procir.2017.03.006 
Kossiakoff, A. and Sweet, W.N. (2003), Systems Engineering: Principles and Practices, Wiley Online Library.

Kulin, M., Fortuna, C., De Poorter, E., Deschrijver, D. and Moerman, I. (2016), "Data-Driven Design of Intelligent Wireless Networks: An Overview and Tutorial", Sensors, Multidisciplinary Digital Publishing Institute, Vol. 16 No. 6, p. 790. https://doi.org/10.3390/s16060790

Kusiak, A. (2006), "Data mining: manufacturing and service applications", International Journal of Production Research, Taylor \& Francis, Vol. 44 No. 18-19, pp. 4175-4191. https://doi.org/10.1080/00207540600632216

Lützenberger, J., Klein, P., Hribernik, K. and Thoben, K.-D. (2016), "Improving Product-Service Systems by Exploiting Information From The Usage Phase. A Case Study”, Procedia CIRP, Vol. 47, pp. 376-381. https://doi.org/10.1016/j.procir.2016.03.064

Ma, H., Chu, X., Lyu, G. and Xue, D. (2017), “An Integrated Approach for Design Improvement Based on Analysis of Time-Dependent Product Usage Data", Journal of Mechanical Design, American Society of Mechanical Engineers Digital Collection, Vol. 139 No. 11. https://doi.org/10.1115/1.4037246

Manyika, J., Chui, M., Institute, M.G., Brown, B., Bughin, J., Dobbs, R., Roxburgh, C., et al. (2011), Big Data: The Next Frontier for Innovation, Competition, and Productivity, McKinsey.

Matthew, N. and Stones, R. (2008), Beginning Linux Programming, John Wiley \& Sons.

Miller, H.G. and Mork, P. (2013), "From Data to Decisions: A Value Chain for Big Data", IT Professional, Vol. 15 , pp. $57-59$.

Nemoto, Y., Akasaka, F., Chiba, R. and Shimomura, Y. (2012), "Establishment of a function embodiment knowledge base for supporting service design", Science China Information Sciences, Vol. 55 No. 5, pp. 1008-1018. https://doi.org/10.1007/s11432-012-4561-3

Poot, L.P., Wehlin, C., Tarkian, M. and Ölvander, J. (2020), "Integrating Sales and Design: Applying CAD Configurators in the Product Development Process", Proceedings of the Design Society: DESIGN Conference, Vol. 1, Cambridge University Press, pp. 345-354.

Rocca, G.L. (2012), "Knowledge based engineering: Between AI and CAD. Review of a language based technology to support engineering design", Advanced Engineering Informatics, Vol. 26 No. 2, pp. 159-179. https://doi.org/10.1016/j.aei.2012.02.002

Sakao, T., Hara, T. and Fukushima, R. (2020), "Using Product/Service-System Family Design for Efficient Customization with Lean Principles: Model, Method, and Tool”, Sustainability, Vol. 12 No. 14, p. 5779. https://doi.org/10.3390/su12145779

Sakao, T. and Neramballi, A. (2020), “A Product/Service System Design Schema: Application to Big Data Analytics”, Sustainability, Vol. 12, p. 3484. https://doi.org/10.3390/su12083484

Sala, R., Bertoni, A., Pirola, F. and Pezzotta, G. (2020), "The Data-Driven Product-Service Systems Design and Delivery (4DPSS) Methodology", presented at the IFIP International Conference on Advances in Production Management Systems, Springer, pp. 314-321.

Shin, J.-H., Kiritsis, D. and Xirouchakis, P. (2015), "Design modification supporting method based on product usage data in closed-loop PLM", International Journal of Computer Integrated Manufacturing, Taylor \& Francis, Vol. 28 No. 6, pp. 551-568. https://doi.org/10.1080/0951192X.2014.900866

Sokolova, M. and Lapalme, G. (2009), "A systematic analysis of performance measures for classification tasks", Information Processing \& Management, Vol. 45 No. 4, pp. 427-437. https://doi.org/10.1016/j.ipm.2009.03.002

Song, W. and Sakao, T. (2017), “A customization-oriented framework for design of sustainable product/service system”, Journal of Cleaner Production, Vol. 140, pp. 1672-1685. https://doi.org/10.1016/j.jclepro.2016.09.111

Stokes, M. and MOKA Consortium. (2001), Managing Engineering Knowledge: MOKA: Methodology for Knowledge Based Engineering Applications, Professional Engineering Publishing.

Sundin, E., Lindahl, M., Comstock, M., Sakao, T. and Shimomura, Y. (2009), “Achieving mass customisation through servicification", International Journal of Internet Manufacturing and Services, Vol. 2, pp. 56-75.

Tarkian, M., Persson, J., Ölvander, J. and Feng, X. (2012), "Multidisciplinary Design Optimization of Modular Industrial Robots by Utilizing High Level CAD Templates", Journal of Mechanical Design, American Society of Mechanical Engineers Digital Collection, Vol. 134 No. 12. https://doi.org/10.1115/1.4007697

Ulrich, K.T. and Eppinger, S.D. (2012), Product Design and Development, Fifth., McGraw-Hill, New York, NY 10020.

van der Velden, C., Bil, C. and Xu, X. (2012), “Adaptable methodology for automation application development”, Advanced Engineering Informatics, Vol. 26 No. 2, pp. 231-250. https://doi.org/10.1016/j.aei.2012.02.007

Whyte, W.F. (1989), “Advancing scientific knowledge through participatory action research”, Sociological Forum, Vol. 4 No. 3, pp. 367-385. https://doi.org/10.1007/BF01115015

Wright, L. and Davidson, S. (2020), "How to tell the difference between a model and a digital twin", Advanced Modeling and Simulation in Engineering Sciences, Vol. 7 No. 1, p. 13. https://doi.org/10.1186/s40323-02000147-4 